\title{
Electromagnetic Field Effect and Analysis of Composite Structure
}

\author{
Kuo-Ta Hsieh and Jerome T. Tzeng
}

\begin{abstract}
The electromagnetic and thermal response of composites subjected to magnetic fields is simulated by solving Maxwell and heat transfer equations simultaneously. The developed analysis accounts for the anisotropic nature of the electrical and thermal properties in three dimensions. A finite element code is developed to predict the response of composite structures subjected to transient magnetic fields. The analysis has been validated against a closed form solution and applied to simulate the induction heating process of composite cylinders. The developed analysis can be applied to the design of modern electrical weapons and used to simulate composite manufacturing processes such as induction cure.
\end{abstract}

\section{INTRODUCTION}

Carbon fiber reinforced composite will respond to an electromagnetic field resulting in a Joule heating in the structure $[1,2]$. The generated heat due to electrical induction may cause composite degradation. There is further need to understand and quantify this heating process in the presence of high strength magnetic fields. The induction heating process has also been successfully used for composite curing and manufacturing in Army's applications. The induction cure process is typically developed based on an empirical or trial and error approach. The process is time consuming and not cost effective. In additional, the optimal process cycle cannot be achieved by using such empirical methods.

The Joule heating and heat transfer in the composite using induction curing processes are extremely anisotropic due to the carbon fiber orientation and distribution. In addition, the strength and frequency of transient electromagnetic field have a great influence on the final quality of the composite. This investigation seeks to develop a computational model and code that can simulate the heating process of a composite structure subjected to transient magnetic fields and simulate the cure kinetics due to induction curing during composite manufacturing. A full set of three dimensional Maxwell equations has been solved using the finite element methods. Electromagnetic analysis is coupled with heat transfer and cure kinetics for modeling composite manufacturing. The model accounts for three dimensional transient electromagnetic field and electrical conductivities and illustration of the numerical results are given in this paper.

Kuo-Ta Hsieh, the University of Texas at Austin, Austin, TX 78759, email:k.hsieh@cem.utxas.edu

Jerome T. Tzeng, US Army Research Laboratory, Aberdeen Proving Ground, MD 21911, USA, e-mail: Jerome.t.tzeng.civ@mail.mil

\section{THEORETIC FORMULATION}

Electromagnetic fields are governed by Maxwell's equations that are basically wave equations. For lower frequency applications, the wavelength of the magnetic field is much longer than the length scale of the systems of interest. In this case, the displacement current can be neglected and the quasi-static approximation is used to describe the diffusion process in place of the wave equations. The magnetic vector potential and electrical scalar potentials are often used to reduce the degree of freedom from six to four for numerical modeling efficiency. The Lagrangian form of magneto-quasistatic diffusion equations (1) and (2) in terms of these dual potentials is implemented in EMAP3D [3]. EMAP3D is a parallel coupled (electromagnetic, thermal, mechanical) physics code that is sponsored by the Army.

Lagrangian Form of Magneto-Quasistatic Diffusion Equations

$$
\begin{aligned}
& \sigma(\mathrm{T}) \frac{\partial \vec{A}(\vec{X}, t)}{\partial t}+\nabla \times\left\{\frac{1}{\mu(|\vec{B}|)} \nabla \times \vec{A}(\vec{X}, t)\right\}+\sigma(\mathrm{T}) \nabla \varphi(\vec{X}, t)=0 \\
& \nabla \bullet\left\{-\sigma(\mathrm{T}) \frac{\partial \vec{A}(\vec{X}, t)}{\partial t}-\sigma(\mathrm{T}) \nabla \varphi(\vec{X}, t)\right\}=0 \\
& \vec{B}(\vec{X}, t)=\nabla \times \vec{A}(\vec{X}, t) ; \quad \nabla \bullet \vec{A}(\vec{X}, t)=0 \\
& \vec{J}(\vec{X}, t)=\sigma(\mathrm{T}) \vec{E}(\vec{X}, t) \quad ; \quad \vec{B}(\vec{X}, t)=\mu(\vec{B} \mid) \vec{H}(\vec{X}, t)
\end{aligned}
$$

$$
\begin{aligned}
& \text { Where } \\
& \vec{X} \equiv \text { Material coordinates } \\
& \text { T } \equiv \text { Temperature } \\
& t \equiv \text { Time } \\
& \vec{A}(\vec{X}, t) \equiv \text { Magnetic vector potential } \\
& \varphi(\vec{X}, t) \equiv \text { Electrical scalar potential } \\
& \vec{B}(\vec{X}, t) \equiv \text { Magnetic induction } \\
& \vec{H}(\vec{X}, t) \equiv \text { Magnetic intensity } \\
& \vec{J}(\vec{X}, t) \equiv \text { Current density } \\
& \vec{E}(\vec{X}, t) \equiv \text { Electric field } \\
& \sigma(\mathrm{T}) \equiv \text { Electric conductivity } \\
& \mu(|\vec{B}|) \equiv \text { Permeability }
\end{aligned}
$$

The implementation of the potential formulation can be verified by a 2D problem: eddy current induction in a solid conducting cylinder with a transverse magnetic field that has 
analytic solutions [4]. According to the induction law, the electromotive force (current) can be induced by either the relative motion between the conductors and magnetic fields (i.e. flux cutting) or time varying magnetic fields. This paper will investigate the induced current by time varying (sinusoidal) magnetic field as depicted in figure 1 .

The governing equations for $2 \mathrm{D}$ problems can be described as in equations (3-5). The predicted magnetic inductions are shown in figure 2. The comparison of the $y$ components of calculated magnetic induction with the analytic solutions is shown in figure 3. The results are identical and verify the implementation.

$\nabla \times \vec{H}=\vec{J} \quad ; \quad \nabla \times \vec{E}=-\frac{\partial B}{\partial t}$

2D Problem:

$\overrightarrow{\mathrm{H}}(\mathrm{x}, \mathrm{y})=\mathrm{H}_{x}(\mathrm{x}, \mathrm{y}) \overrightarrow{\mathrm{i}}+\mathrm{H}_{y}(\mathrm{x}, \mathrm{y}) \overrightarrow{\mathrm{j}} \Rightarrow \overrightarrow{\mathrm{J}}(\mathrm{x}, \mathrm{y})=\mathrm{J}_{z}(\mathrm{x}, \mathrm{y}) \overrightarrow{\mathrm{k}}$

Diffusion Equations :

$\frac{1}{\sigma_{z} \mu_{0}} \frac{\partial B_{x}}{\partial t}+\frac{\partial^{2} B_{y}}{\partial y \partial x}-\frac{\partial^{2} B_{x}}{\partial y^{2}}=0$

$\frac{1}{\sigma_{z} \mu_{0}} \frac{\partial B_{y}}{\partial t}-\frac{\partial^{2} B_{y}}{\partial x^{2}}+\frac{\partial^{2} B_{x}}{\partial x \partial y}=0$

where

$\overrightarrow{\mathrm{H}} \equiv$ Magnetic field intensity

$\overrightarrow{\mathrm{B}} \equiv$ Magnetic induction

$\overrightarrow{\mathrm{J}} \equiv$ Current Density

$\overrightarrow{\mathrm{E}} \equiv$ Electric field intensity

$\mu_{0} \equiv$ Permeability for vacuum

$\sigma_{z} \equiv$ Electrical conductivity in z direction

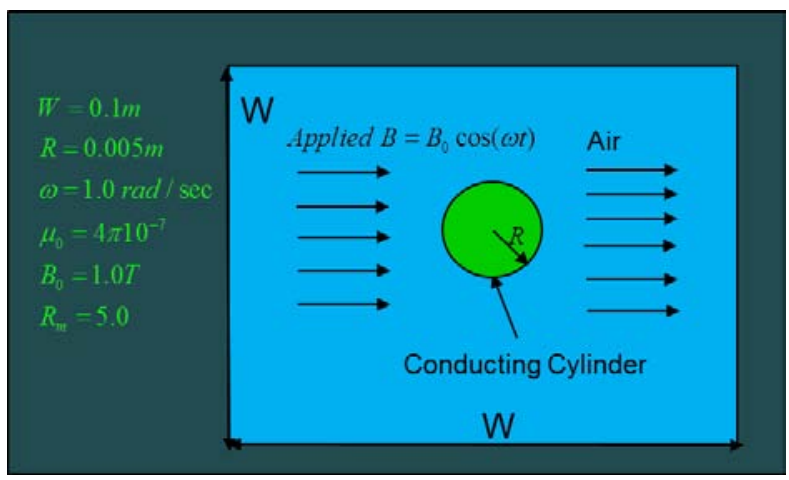

Figure 1: The description of sample problem of a long conducting cylinder subjected to a sinusoidal magnetic field.

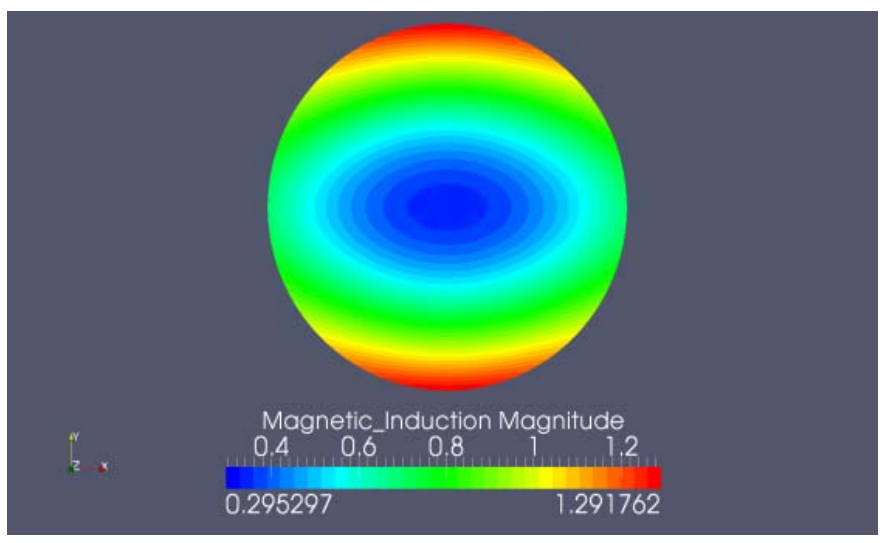

Figure 2: The calculated magnetic induction

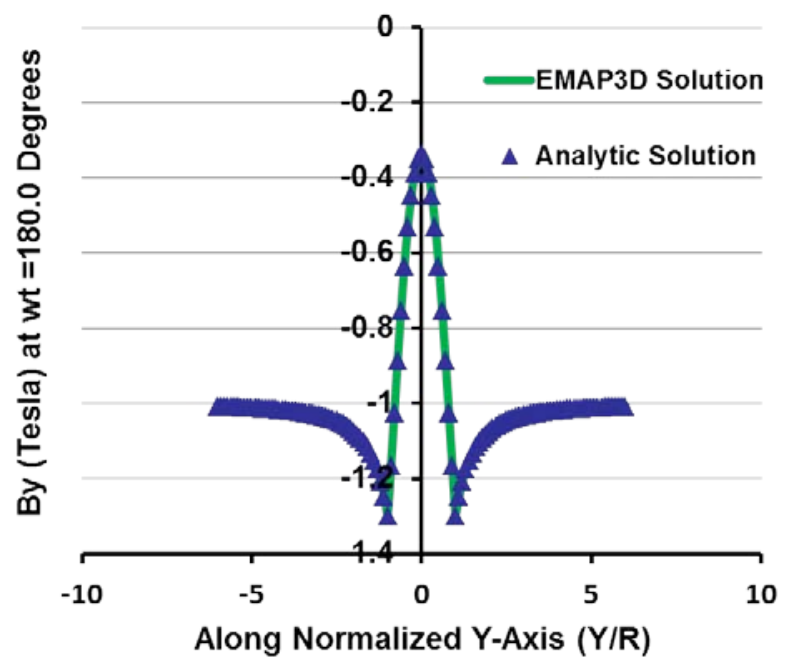

Figure 3: The comparison of calculated y-component of magnetic field with analytic solutions and they are identical.

\section{Heat Transfer in Composites}

Carbon fiber reinforced composite is conductive. Application of an electromagnetic field can result in a significant heating process depending upon the strength and frequency of a transient magnetic field. This process has been recognized in the design and application of modern electromagnetic weapons. The induction heating process can also be applied in composite manufacturing process as a heating mechanism.

Heat transfer and conventional cure kinetics analysis in composite manufacturing has been studied by the author [5] and many researchers previously. The induction heating mechanism can actually be applied in the composite curing process to replace conventional oven or autoclave heating. In fact, the inductive heating cure process (IHCP) has been used in Army 
applications because of its fast, uniform heating and controllable features. In the following analysis, the Joule heating due to induced electrical current is incorporated with heat transfer for modeling the composite curing process. The associated cure kinetics can also be included in the analysis. Thermal equation including curing kinetics is described in equations (5-6).

Thermal Diffusion and Curing Model

$C_{p} \frac{\partial T}{\partial t}=\nabla \bullet(-\tilde{K} \nabla T)+S$

Where

$S \equiv$ Heat source $\equiv S_{c}+S_{i}$

$S_{i} \equiv$ Inductive heat rate $\equiv \vec{E} \bullet \vec{J}$

$S_{i}=0$ for conventional curing process in which heat flux is applied

$\vec{E} \equiv$ Electrical Field

$\vec{J} \equiv$ Current density

$S_{c} \equiv$ Exothermic heat rate due to curing reaction $\equiv \rho_{r} V_{r} H_{r} \frac{d \alpha}{d t}$

$H_{r} \equiv$ Exothermic enthalpy

$\alpha \equiv$ Curing degree

$\rho_{r} V_{r} \equiv$ Mass fraction of resin

$C_{p} \equiv$ Specific heat

$\tilde{K} \equiv$ Thermal conductivity matrix

Curing Kinetics

$\frac{d \alpha}{d t}=f(\alpha, t)$

$f(\alpha, t)=A e^{\left(-\frac{\Delta E}{R T}\right)} \alpha^{m}(1-\alpha)^{n} \quad$ or

$f(\alpha, t)=\left(A_{1} e^{\left(-\frac{\Delta E_{1}}{R T}\right)}+A_{2} e^{\left(-\frac{\Delta E_{2}}{R T}\right)} \alpha^{m}\right)(1-\alpha)^{n}$

Where

$\Delta E \equiv$ Activation energy

$R \equiv$ Universal gas constant

\section{CASE STUDY}

This paper also investigated the induction heating process in composites in various aspects such as 3D effects, the effect of angular velocities, the effect of fiber orientation, and multi-cycle effects. A 3D model of a hollow conducting composite cylinder subjected to a sinusoidal magnetic field of
0.5Tesla was constructed as shown in figure 4 to study these effects. The dimensions of the cylinder are $0.1 \mathrm{~m}$ diameter and $0.05 \mathrm{~m}$ long. The specific heat of the composite is 875 joules per cubic meter. The electrical conductivities of composite are $40,000(\mathrm{ohm}-\mathrm{m})^{-1}$ and $667(\mathrm{ohm}-\mathrm{m})^{-1}$ in the fiber and transverse (orthogonal to fiber) direction, respectively. Two fiber orientations (circumferential and axial directions) are considered. Tensor transformation is then applied according to the orientation of the fiber in the cylinder. The magnetic field is applied in either axial or transverse direction in the following simulations.

The effect of frequency of the magmatic field (a transient sinusoidal field) is illustrated by comparing induction heating of two angular frequencies of $377.0 \mathrm{rad} / \mathrm{sec}$ and 754.0 $\mathrm{rad} / \mathrm{sec}$ for the configuration of transverse magnetic field and axial fiber. Figure 5 shows that a higher angular velocity induced a higher current as expected. The effects on temperature and current flow of angular velocity are shown in figure 6 and figure 7 , respectively. The peak temperatures are $329{ }^{\circ} \mathrm{C}$ and $357{ }^{\circ} \mathrm{C}$, for low and high frequencies respectively. The temperature change is almost double for the high frequency case.

The fiber orientation and layup of the composite cylinder has great influence on the induction heating process since the electrical conductivity of the composite is mainly determined by the carbon fiber. The effect of fiber orientation on the heat generation and temperature rise in the composite cylinder is illustrated in the simulations with axial magnetic field. The fibers oriented in the axial and circumferential directions were simulated to study the effects of fiber orientation shown in Figure 8. An exactly same magnitude of axial magnetic field is applied in both cases. The induced current and temperature are higher for the circumferential oriented fiber as shown in Figure 8. The induction coupling or heating process is significantly larger if the magnetic field is perpendicular to the fiber direction.

For application of a transient cyclic magnetic field, the effect of cycling number on the heating process is also very significant. Figure 9 and figure 10 shows the response of the current flow and temperature rise to the multiple cycles of magnetic field. The peak temperatures at the outer surface of cylinder are 316 and $380^{\circ} \mathrm{C}$ for one cycle and five cycles of application, respectively. The temperature rise is almost proportional to the number of cycle of magnetic field application.

\section{CONCLUSION}

An electromagnetic analysis is developed to account for the anisotropic induction process and electromagnetic properties of composite materials. In addition, the electromagnetic analysis is extended to simulate the induction curing process. In this paper, the electromagnetic analysis is solved using the finite element technique and is validated by comparison to a closed-form solution. Case studies were performed to show the effect of the 
magnetic field, fiber orientation and number of cycles on the heating process in a conducting composite cylinder. A cure kinetics is also formulated and integrated into the developed analysis for composite fabrication simulation.

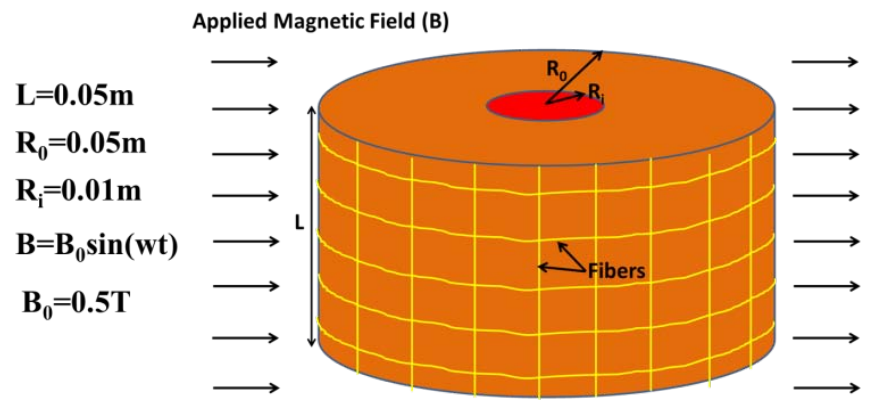

$f=60 H_{z} ; w=2 \pi f=377 \mathrm{rad} / \mathrm{s} ; T=\frac{1}{f}=0.0167 \mathrm{sec}$

$f=120 H_{z} ; w=2 \pi f=754 \mathrm{rad} / \mathrm{s} ; T=\frac{1}{f}=0.00833 \mathrm{sec}$

Figure 4: 3D model of a conducting composite cylinder subjected to a sinusoidal magnetic field

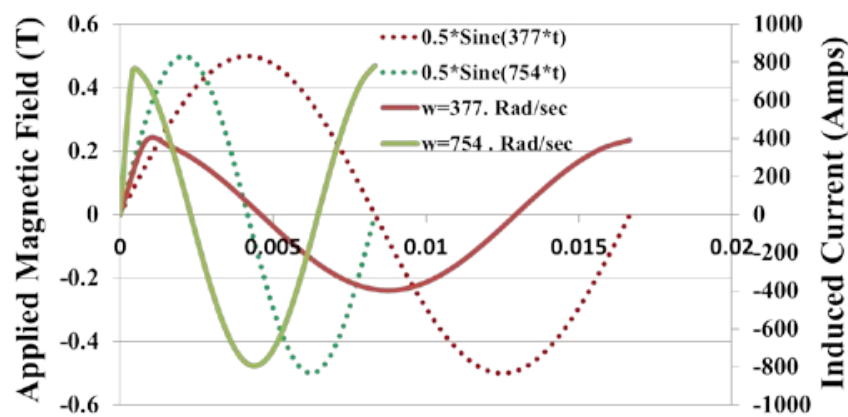

Time (sec)

Figure 5: The effects of the angular velocity on the profiles of calculated induced currents.

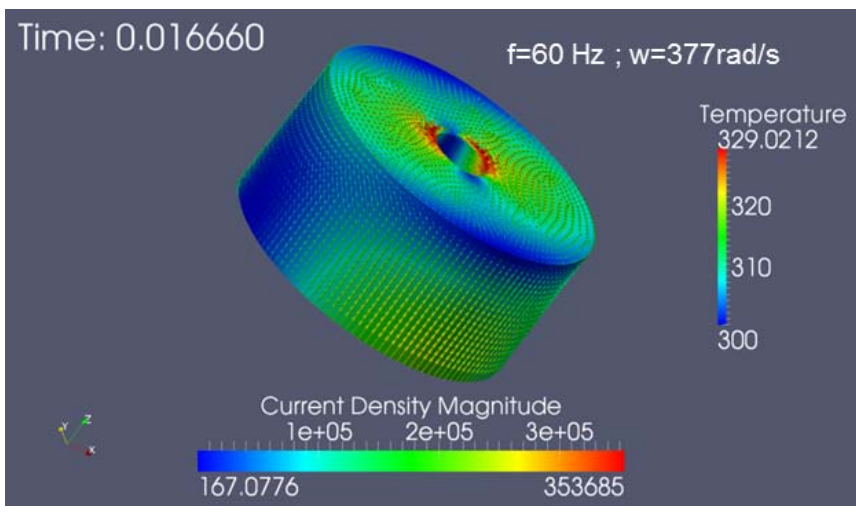

Figure 6: Temperature and current flow for the $377.0 \mathrm{rad} / \mathrm{sec}$.

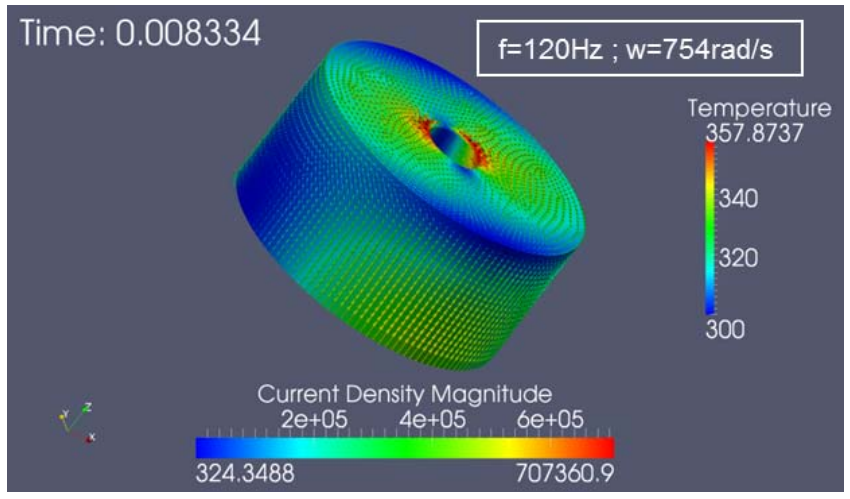

Figure 7: Temperature and current flow for the $754.0 \mathrm{rad} / \mathrm{s}$

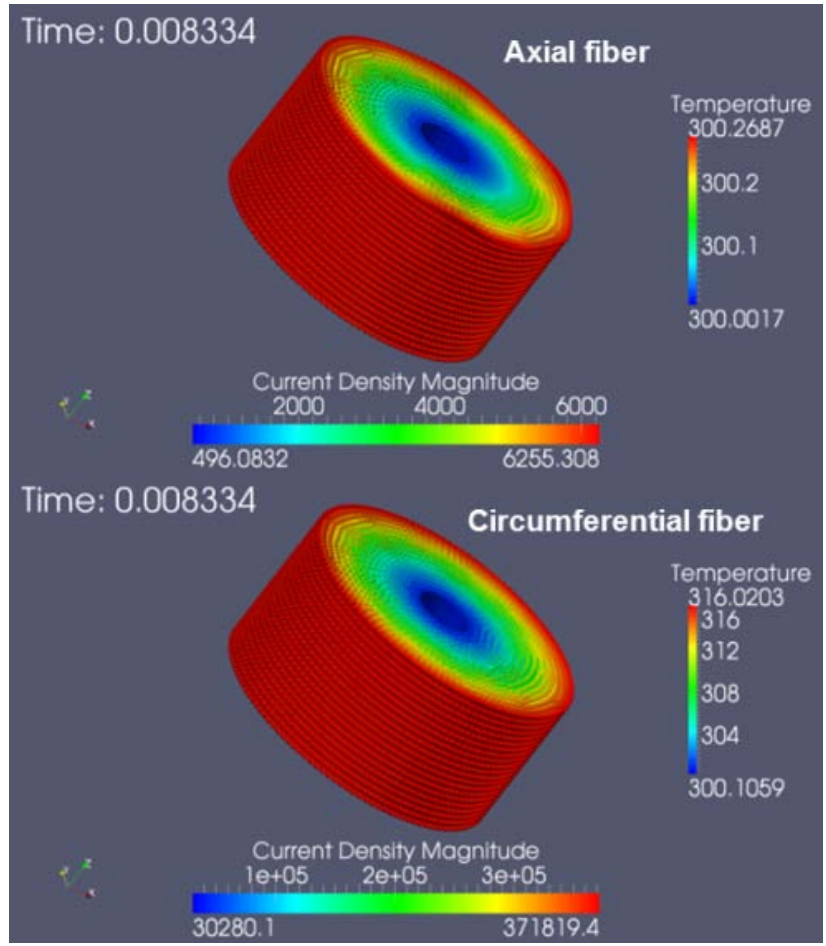

Figure 8: The effects of fiber orientations on temperature and current flow. 


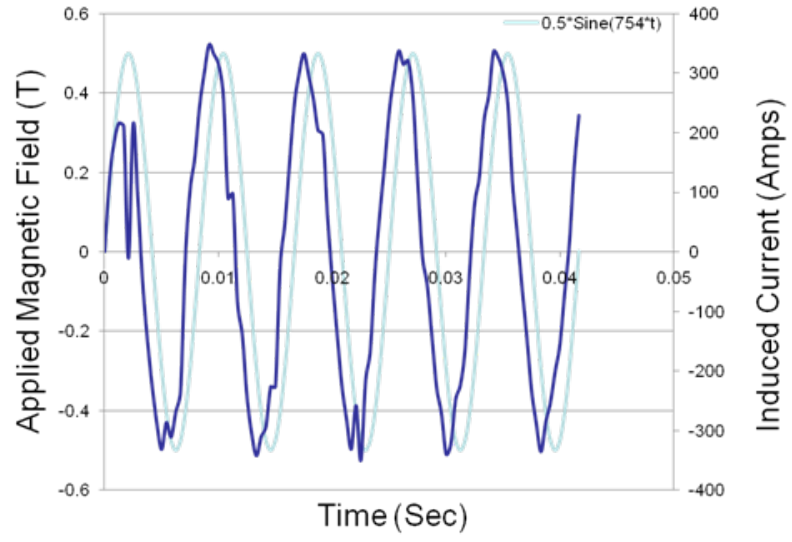

Figure 9: Calculated induced current history under five cycles of applied magnetic fields.

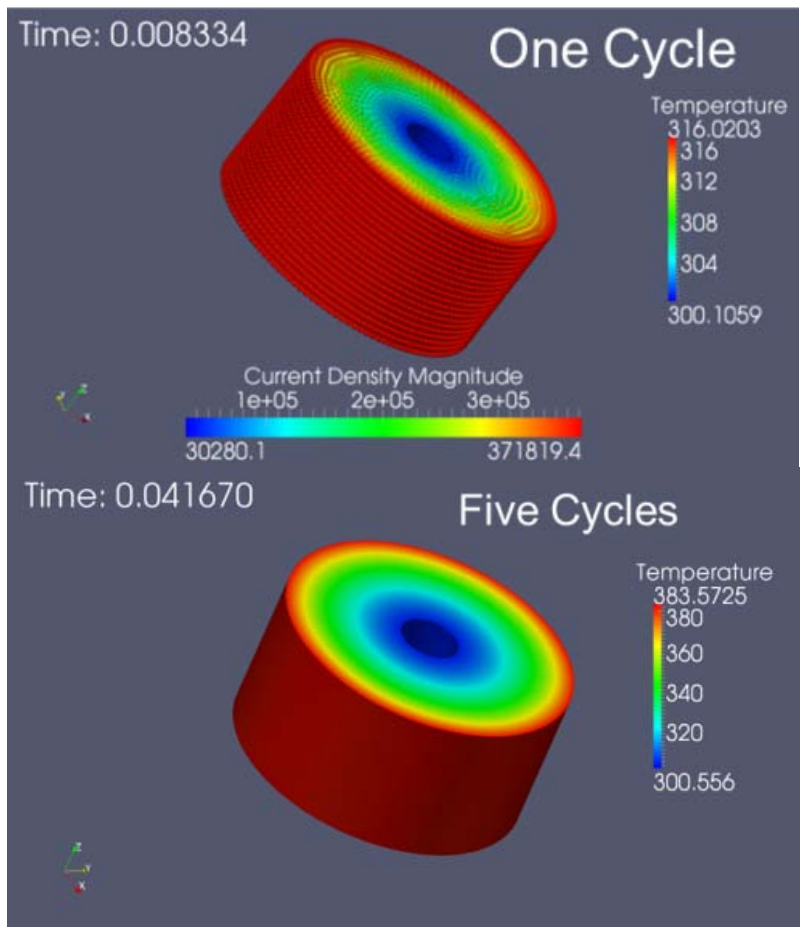

Figure 10: Temperature and current flow for one cycle and five cycles of excitations.

\section{REFERENCE}

1. B. K. Fink, R.L. McCullough, and J.W. Gillespie, "Induction Heating of Carbon-fiber Composites: Electrical Potential Distribution Model, ARL-TR-2130, Army Research Laboratory, Aberdeen Proving Ground, MD, Nov. 1999.
2. H. Kim, S. Yarlagadda, J.W. Gillespie, N.B. Shevehenko, B.K. Fink, "A Study on Induction Heating of Carbon Fiber Reinforced Thermoplastic Composites," Journal of Advanced Composite Materials, Vol 11, Issue 1, 2002.

3. K. T. Hsieh, "A Lagrangian Formulation for Mechanically, Thermally Coupled Electromagnetic Diffusive Processes with Moving Conductors,” IEEE Trans on Magnetics, vol. 31, No. 1, 1995, pp. 604-609.

4. M.P. Perry and T.B. Jones; "Eddy Current Induction in a Solid Conducting Cylinder with a Transverse Magnetic Field," IEEE Transactions on Magnetics, vol.MAG-14, No. 4, July 1978, pp. 227-232.

5. Tzeng, J. T. and A. L. Loos, "A Cure Analysis for Axisymmetric Composites," Journal of Composite Manufacturing, Vol 4, No 3, 1993, pp. 157-165. 


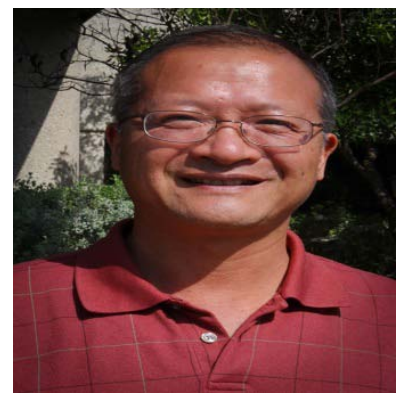

Dr. Hsieh joined Center for Electromechanics at the University of Texas at Austin (UT-CEM) in 2012 as a senior research scientist. His primary research interest has been on parallel multiphysics modeling and simulation e.g. modeling of high power semiconductor devices. Prior joining UT-CEM, Dr. Hsieh was an associate director at UT-IAT where he leaded the Material and Computational Analysis Division. The group focuses on electromagnetic fracture mechanics, tribology of highspeed electrical contacts, the characterization of material behavior under high strain rate and high heating conditions and EM effects on conductive fibrous composites. Dr. Hsieh has developed a state-of-the-art 3D finite element code, EMAP3D for solutions of coupled electromagnetic / chemical / thermal / mechanical equations and has established and operated a parallel computing facility. Dr. Hsieh has more than 100 technical publications.

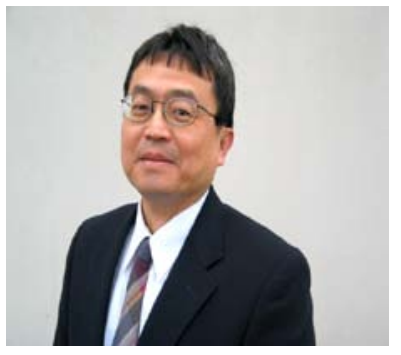

Dr. Jerome T. Tzeng has worked at U.S. Ballistic Research Laboratory/Army Research Laboratory since 1991. Prior to joining the government, he worked with DuPont as a research engineer and Bechtel Corporation as a structural engineer. He received his $\mathrm{PhD}$ in Engineering Mechanics from Virginia Tech and Master in Civil Engineering from Rice University. Dr. Tzeng works in the area of composite materials for ballistic applications such as advanced projectiles and gun systems. His current research has concentrated on the vehicle crew protection and advanced gun technology such as electromagnetic rail guns including pulsed power and advanced conventional guns and projectiles. Dr. Tzeng is also actively involved in the basic research, as a journal editor and an adjunct professor. He has more than 100 technical publications in various areas. 